\title{
Implementing a high performance AA-mid in SKA2
}

\author{
Andrew Faulkner ${ }^{1}$ \\ University of Cambridge \\ Cavendish Laboratory, JJ Thompson Avenue, Cambridge, UK \\ E-mail: ajf@mrao.cam.ac.uk
}

\begin{abstract}
Aperture phased arrays operating at mid-frequencies from $400 \mathrm{MHz}$ to $1.4 \mathrm{GHz}$ are a very exciting receiver technology being developed for the Square Kilometre Array, SKA. In many respects they enable the original concept of the SKA: to survey hydrogen in the universe out to a high redshift. The capabilities of a telescope using phased array stations brings major scientific opportunities, including very high survey speeds, flexibility for searching for transient phenomena, and multi-beaming capability which cannot be realized using any other technology. The very high level of control over each receiver element enables exquisite calibration as a function of frequency and pointing direction. The technological requirements to implement high frequency, astronomically capable phased arrays are severe in terms of power and cost due to the large numbers of channels and the amount of digital processing required. However, technology roadmapping shows that forthcoming technical developments make a large system deployment realistic from 2020 onwards. An aperture array covering this frequency range is the only instrument able to perform some of the most challenging science experiments planned for the SKA and is likely to make transformational discoveries.
\end{abstract}

Resolving the Sky - Radio Interferometry: Past, Present and Future

Manchester, UK

April 17-20, 2012

\footnotetext{
$1 \quad$ Speaker
} 


\section{Introduction}

The Square Kilometre Array, SKA is the next generation low frequency radio telescope with preliminary specifications described by Schilizzi et al. [1]. The work performed in the European funded FP6 programme, SKA Design Studies, SKADS, [2] showed that an implementation of the SKA using phased aperture arrays, AAs, operating from $70 \mathrm{MHz}$ up to 1.4GHz with a dish based array covering higher frequencies represents the most capable design for the SKA Phase 2 science case [3]. Here we discuss the scientific requirements of a midfrequency AA and the need for the proposed frequency range; the development and some tradeoffs of a suitable array.

The deployment of the SKA, will start with a 10\% instrument, Phase 1, in 2016 which includes a low frequency, sparse AA covering $70 \mathrm{MHz}-450 \mathrm{MHz}$, the details are described in SKA memo 125 [4]. The development of the much more technically challenging dense AA-mid system will continue in parallel, preparing for deployment in SKA Phase 2 commencing in the early 2020s. This schedule enables SKA Phase 1 to benefit from the experience gained with current low frequency AAs, LOFAR [5] and MWA [6] systems, followed by the more difficult dense AA in Phase 2.

\section{Scientific considerations}

An AA is a very different receiver concept from conventional dishes; it has multi-beaming capabilities, with very fast change in observation direction capability. The SKA Design Reference Mission [3], is roughly analysed in Figure 1 showing the sensitivity and survey speed requirements of the various science experiments. Most of the major surveys are performed at frequencies below the neutral hydrogen line at $1.4 \mathrm{GHz}$; this sets the highest frequency required of the AA system.

Remote galaxies are receding progressively faster due to the expansion of the Universe, this Doppler shifts the hydrogen line to lower frequencies, which enables measuring a three dimensional structure, however, the signal also gets progressively weaker and increases the survey speed requirement at lower frequencies, it is the case that the FoV needs to increase as $\sim \lambda^{3}$ for an optimum survey time. An AA can adjust survey speed as a function of frequency by varying the number of beams across the bandwidth.

The requirement for high dynamic range imaging, particularly for continuum experiments exceeding $10^{7}: 1$, will be very difficult to achieve. AA's have the characteristics necessary to achieve excellent dynamic range: physical stability, unblocked aperture, small individual beams due to the large diameter of the array, ability for exquisite calibration of the "surface" over frequency. There is substantial research ongoing into optimizing the appropriate calibration techniques.

Pulsar and transient detections require a large number of beams for fast surveys and timing of multiple pulsars concurrently; AAs also have the ability to buffer data which enables a "look back" to find the pre-cursor of a transient event. 


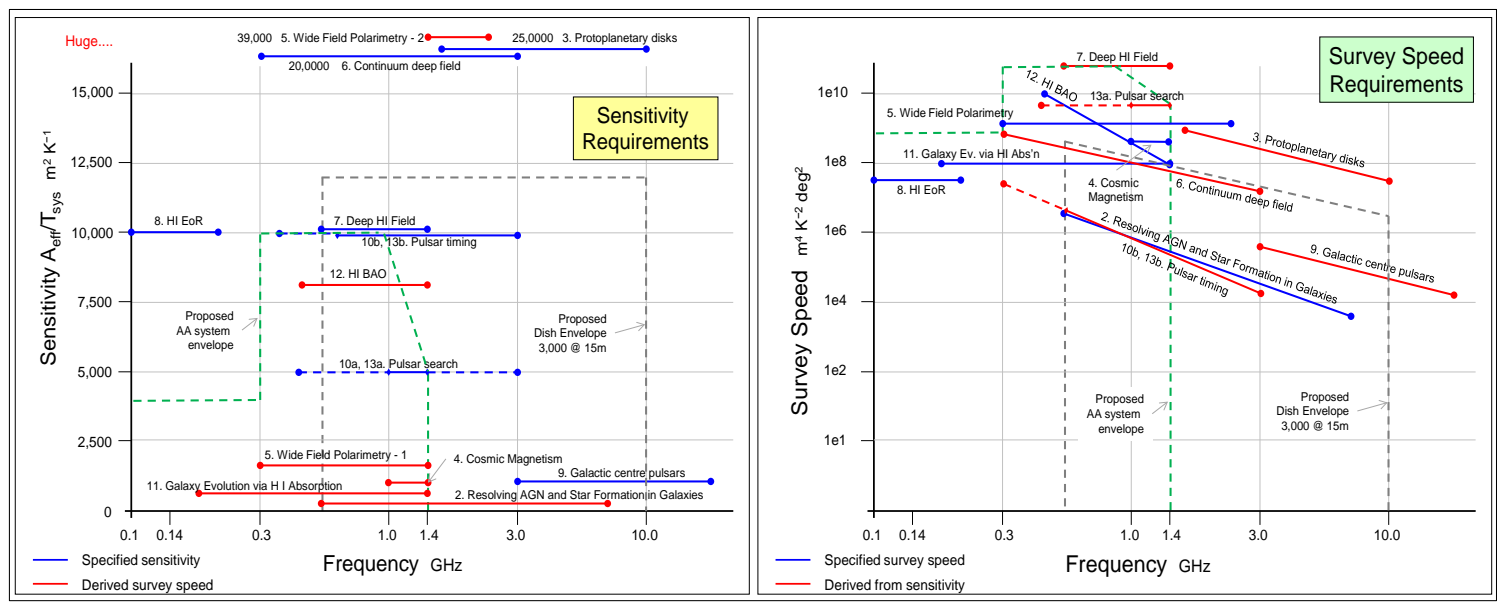

Figure 1: The sensitivity and survey speed requirements for the SKA science case. The sensitivity specifications are shown in the left hand panel with the survey speed's on the right. Note that high survey speeds are typically needed below $1.4 \mathrm{GHz}$.

\section{Aperture array configurations}

Aperture arrays have two main variants: "dense” apertures arrays which have elements spaced $<\lambda / 2$ apart and "sparse" which uses element spacing $>\lambda / 2$. Their characteristics are different and the SKA will use both types as illustrated in Figure 2.

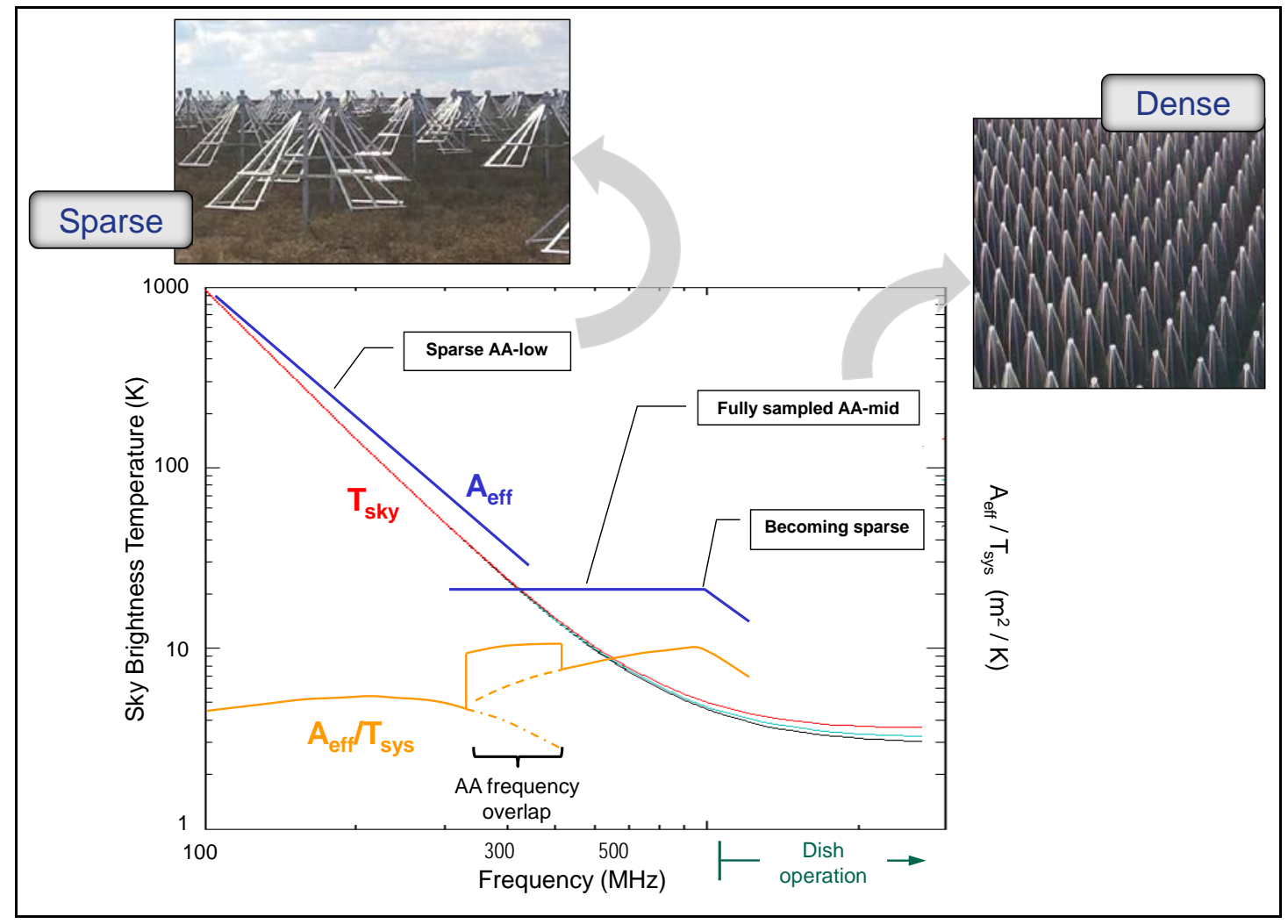

Figure 2: Illustration of the choice of sparse and dense arrays. Sparse arrays are used at low frequency to counter the increasing sky noise with increasing wavelength and dense arrays at the mid-frequencies where sky noise is relatively stable. 
The sparse elements have the characteristic of increasing their effective collecting area as $\lambda^{2}$, which helps to mitigate the effects of increasing sky noise. In the frequency range 400$1400 \mathrm{MHz}$ sky noise is relatively stable and a dense array is selected. This has the ability to fully sample the incoming electromagnetic wavefront and deliver potentially higher dynamic range images. The dense array has essentially a constant effective collecting area, with elements oversampling at the lower operating frequencies of the array.

\section{Dense array design}

The SKA dense phased array, illustrated in Figure 3, consists of a fixed, regular array of small receiving elements each of which are connected to a low-noise amplifier, filtered and further amplified; signals from groups of elements, are "beamformed" using either digital techniques or in the analogue domain and digitized. Beamforming consists of using appropriate time delays on the individual element signals to align their arrival time at a summing point to form a coherent beam in a specific direction. The signals are calibrated in terms of amplitude and phase, equivalent to aligning a parabolic dish surface, except that an AA can also be calibrated as a function of frequency. A number of "Tiles" of elements are arranged into an AA station; the beamformed signals from each Tile are then combined in the station signal processors to further beamform the "Tile beams" into a large number of "Station beams" to cover a sufficient area of the sky for the required survey speed.

Electromagnetic coupling between the elements enables the array to operate at low frequencies, albeit with spatial oversampling. At wavelengths shorter than $\lambda / 2$ the array becomes "sparse" with grating lobes appearing and reducing sensitivity, this is a useful technique to extend the upper frequency within a cost budget. The number of elements required is a square law of the highest dense frequency and strongly determines the total cost and power requirements of the array. The high frequency is therefore critical for the optimum science delivery within a budget.

The performance benefits of dense aperture array are because the incoming wavefront is fully sampled, if each polarization for every element is digitized then the array can be fully calibrated spatially for phase, gain and polarization as a function of frequency. This is a major advantage for dense AAs, but also a challenge in developing the software algorithms and calibration techniques required.

The total array output bandwidth, a sum of the bandwidth of every beam formed, determines the capability of the array to perform surveys or the number of simultaneous observations. The outline specification for the dense AA in the SKA is shown in Table 1. It is an extremely challenging set of specifications which will require projected technology developments to achieve. 
Table 1: Outline SKA AA-mid.

\begin{tabular}{|lr||lr|}
\hline Frequency range: & $400-1450 \mathrm{MHz}$ & No. of elements per array: & $\sim 110,000$ \\
\hline Nyquist frequency: & $1000 \mathrm{MHz}$ & Array diameter: & $56 \mathrm{~m}$ \\
\hline Number of arrays: & 250 & Array output data rate: & $16 \mathrm{~Tb} / \mathrm{s}$ \\
\hline Sensitivity at 800MHz: & $10,000 \mathrm{~m}^{2} / \mathrm{K}$ & Element pitch: & $15 \mathrm{~cm}$ \\
\hline Scan angle range: & $\pm 45^{\circ}$ & Cluster size: & 4 elements \\
\hline System temperature: & $<40 \mathrm{~K}$ & Tile size: & $16 \times 16$ elements \\
\hline Field of View: & $\sim 200 \mathrm{deg}^{2}$ & No. of Tiles per array: & 430 \\
\hline Dynamic range: & $>10^{7}: 1$ & Beamforming technology & Digital \\
\hline Configuration: & $60 \%$ within $5 \mathrm{~km}$ & Digitisation rate: & $3 G$ Gamples $/ \mathrm{s}$ \\
\hline Max. baseline: & $180 \mathrm{~km}$ & Digitisation depth: & 6 -bit \\
\hline
\end{tabular}

\section{AA-mid design considerations}

A dense AA station for the SKA has of the order of 110,000 dual polarization elements; this implies a large amount of electronics which has severe cost and power implications. The array has effectively a fixed collecting area. Therefore the array sensitivity will be dominated by receiver noise giving the requirement of the lowest possible front-end noise performance for good sensitivity and reduced costs. The top frequency is limited by the number of elements that can be afforded; to stretch to $1.4 \mathrm{GHz}$ the system becomes sparse above $1 \mathrm{GHz}$.

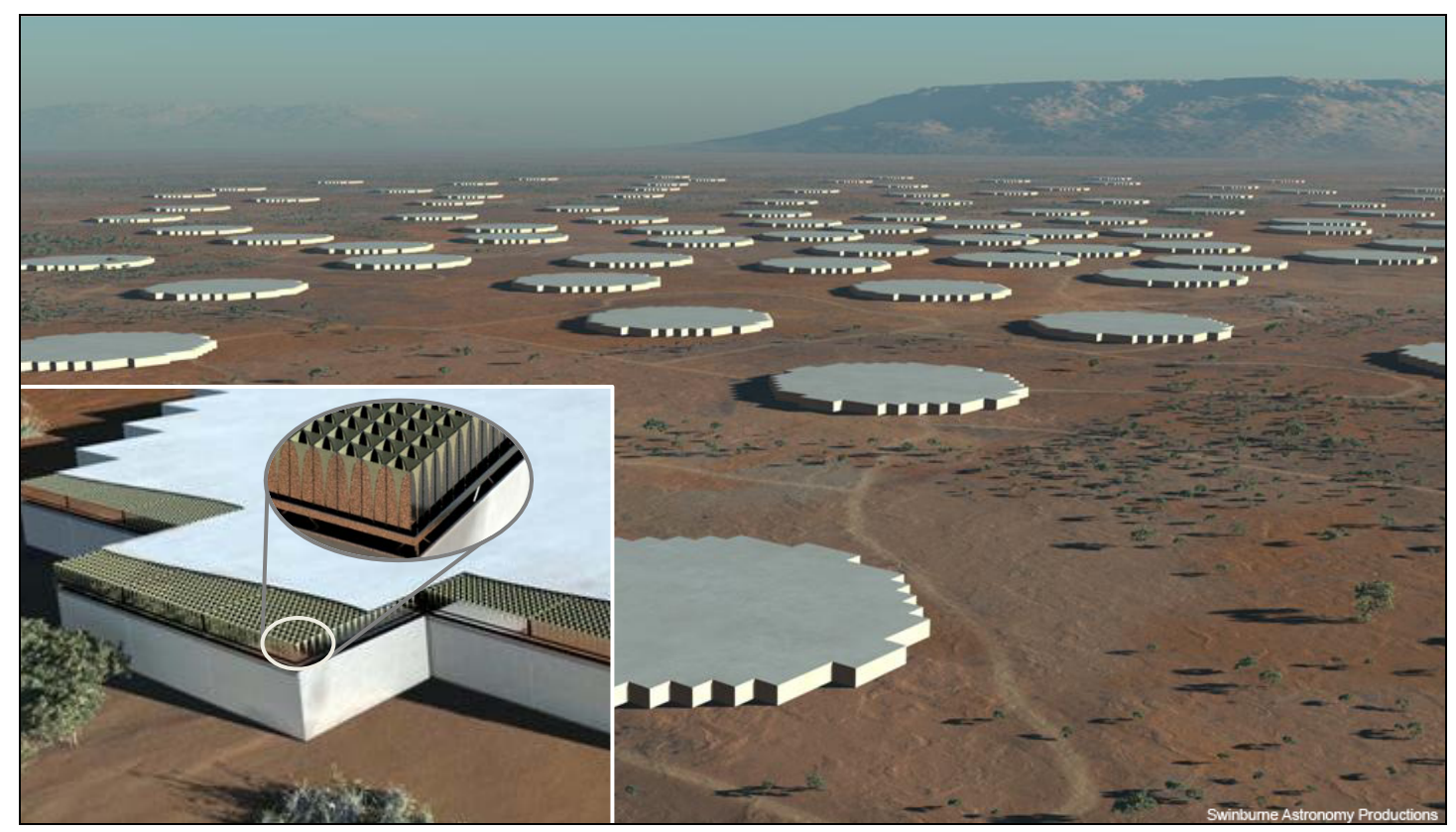

Figure 3: An illustration of AA-mid in the SKA. There are many AA-mid stations each $\sim 56 m$ diameter. Inset shows the dense element pattern that makes up the arrays.

An important decision for the array system design is how many elements will form a "cluster" to be beamformed using analogue techniques prior to using digital processing. Analogue beamforming is cheaper certainly today than a digital system, but has some significant limitations: each beam that is formed requires another set of hardware; it is hard to have precise calibration, particularly to correct polarization issues; and analogue systems have 
potential drift issues. Digital signal processors, DSP, can implement high precision calibration and beamforming, and can provide a large number of beams just by using additional processing and communications capability. Analogue beamforming could use true time delays, TTDs, e.g. using circuit board tracks, but these are large; alternatively, phase shifters can be used, since they are easier to integrate, but they are relatively narrow band because low frequencies are delayed more than high frequencies, preventing a full bandwidth coherent beam. The ideal architecture is to digitize every element path and perform all the beamforming in the digital domain. This is very flexible; however, it is currently more expensive and higher power than an analogue system. The expectation is for a 2020 implementation to use the signal path shown in Figure 4, using TTD for small clusters of elements, probably four, and digitize the single, very large, beam that is produced. DSPs then form a very large number of beams from the array, within a field of view defined by the analogue beam.

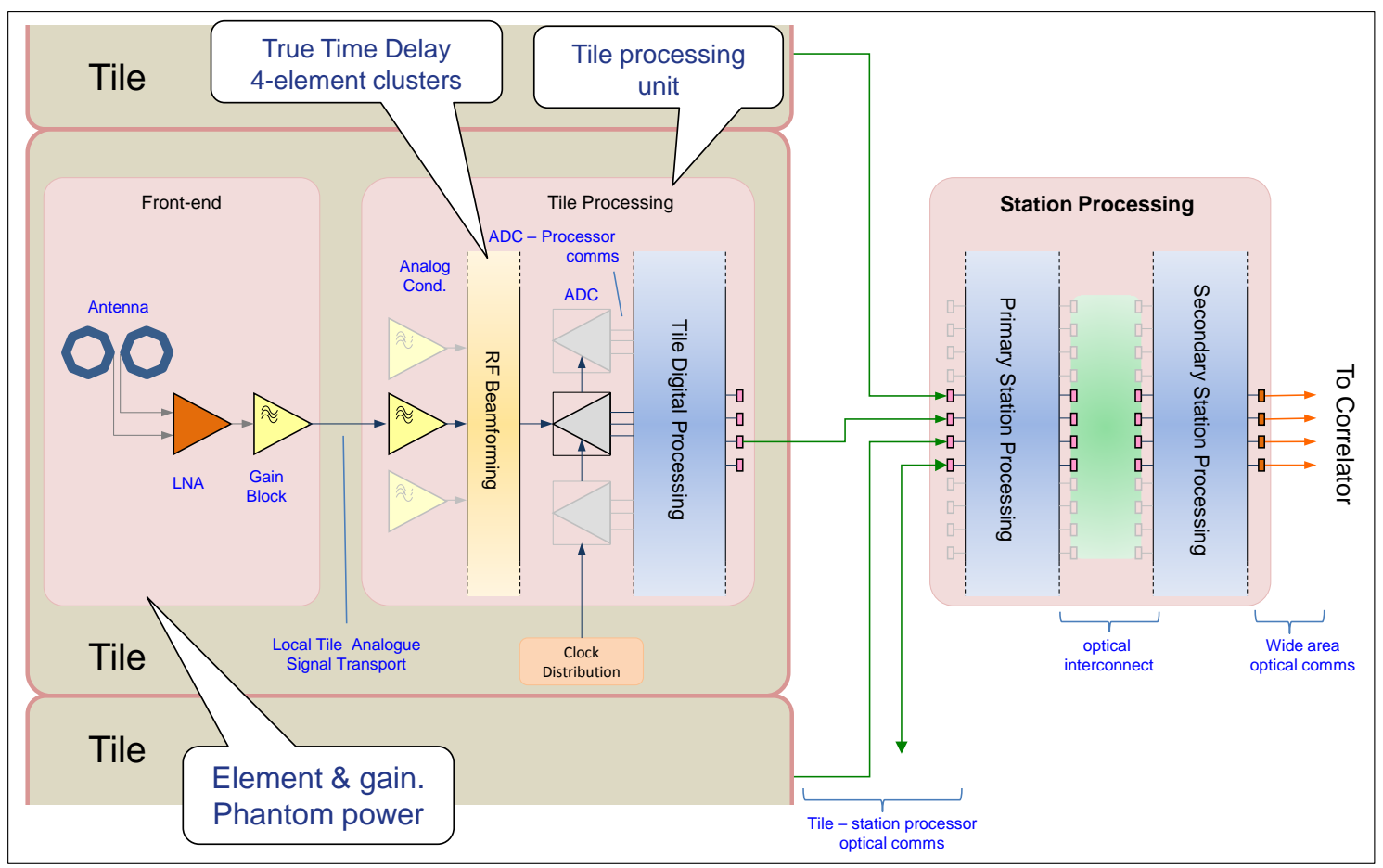

Figure 4: AA-mid signal path showing element LNAand gain, TTD beamforming, digitisation and station beamforming using Tile and station digital signal processing.

The maximum instantaneous bandwidth of the system is the full frequency range that the array operates over. There may be some restrictions, discussed above; however the more important consideration is the total data rate from the array, which ultimately defines the performance of the system. With a flexible system, which is ideal for maximizing the science output, beams will not necessarily need to form conventional beams, but can be tailored over the observed sky as a function of frequency; for example to cover a relatively narrow frequency band over a larger observed sky area e.g. for transient event search; or create a constant field of view independent of frequency.

The sensitivity of the system is a function of frequency and is determined from: size and number of arrays, system temperature $\left(\mathrm{T}_{\text {sys }}\right)$, scan angle and the apodisation employed. A critical 
factor is the receiver noise, dominated by the front-end: element, first amplifier and their matching. Due to the number of receivers and the physical size of the array it is not practical to consider cooling the front-ends for improved noise figures. Consequently, the system will be running at ambient temperature. There had been a lot of progress in this area and the current best front-ends have a $\mathrm{T}_{\text {sys }}$ of $<60 \mathrm{~K}$ (including sky and receiver noise), with $<50 \mathrm{~K}$ expected in 2011, as can be seen in Table 1 , we require $<40 \mathrm{~K}$, which is expected to be achieved before 2016.

Because an AA is essentially a major processing system with receiver inputs, the dynamic range and polarization purity requirements of the SKA are achievable, but will require sufficient analogue stability, and use an array of sufficient diameter to measure and counter the atmospheric effects. Only the required number of arrays for imaging quality will be deployed, the central processing requirements are thus reduced to a reasonable level considering the survey speeds and data rates that are achieved. In effect the processing in the arrays is mitigating the central processing load.

\section{AA-mid construction}

There is considerable work to be done before a definitive mechanical design for AA-mid can be implemented, however, some general design points can be made. Major considerations are: the need to make the element spacing constant, over the whole array, effectively a sea of elements; appropriate interconnect between Tiles; ease of construction and maintenance; low cost; controlling the environment around the electronics and longevity. An illustration is shown in Figure 5.

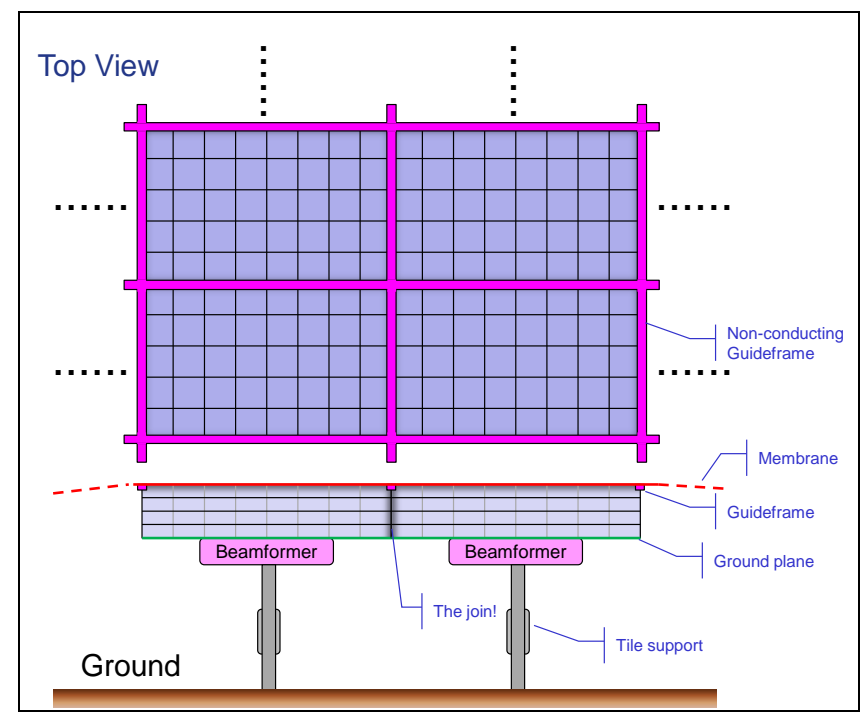

Figure 5: Illustrative mechanical design for AA-mid.

The elements are built into Tiles that connect between themselves for the elements, each Tile has integral electronics and delivers the first stage of process delivering beams over fibre back to the station processing. The Tiles are held up above the ground on a single post and located using a guidframe over the top; Tiles may then be extracted by dropping them down and wheeling them out out of the array between supporting posts. 


\section{Technology Roadmap}

The technologies required for the AAs are generally the focus of the ICT industry: faster and lower power processing, higher speed communications, lower cost, increased storage etc. Hence, AAs tend over time to get more practical, with better performance. At some time, dense AAs become affordable and indeed cheaper than traditional dishes. By carefully studying technology roadmaps for fabrication capabilities, discussion with major semiconductor and communication companies and review of markets that require similar components we anticipate that a dense AA system for the SKA meeting performance, cost and power constraints can be scheduled for 2018 construction.

The key components for the array itself, not currently available, are: an LNA with $<15 \mathrm{~K}$ noise and an analogue system with $<150 \mathrm{~mW}$ total power; a DSP, chip providing >20TMACs and use 25 watts integrated with up to 128 6-bit 3GS/s digitizers each using $<100 \mathrm{~mW}$; a programmable DSP of $>20$ TMACS with $128 \mathrm{I} / \mathrm{Os}$ of $>10 \mathrm{~Gb} / \mathrm{s}$ each; and short-range $\sim 50 \mathrm{~m}$ pluggable optical links of $>120 \mathrm{~Gb} / \mathrm{s}$ with $>2.5 \mathrm{~W}$ power. There is a similar requirement list for the central processing systems. These are all projected and indeed will improve post 2020.

\section{Conclusions}

If dense AAs can be implemented within the cost and power constraints in the timescale of the SKA they are the most capable technology available, representing almost the perfect collector system. The research performed in the SKADS program shows that technology evolution will be sufficient to enable substantial deployment in the second phase of the SKA starting in 2020.

\section{Acknowledgments}

This paper is due to the work of many people in the SKADS project and continuing through the Aperture Array Verification Programme. The author does not wish to list just a few participants, but to fully acknowledge the contributions made by everyone.

\section{References}

[1] Schilizzi R. et al., "Preliminary Specifications for the Square Kilometre Array”, 2007, www.skatelescope.org

[2] “Square Kilometre Array Design Studies, SKADS”, www.skads-eu.org

[3] A. J. Faulkner et al., "SKA Memo 122: Aperture Arrays for the SKA - the SKADS White Paper", 2010

[4] M. A. Garrett et al., "SKA Memo 125: A Concept Design for SKA Phase 1 (SKA1)”, 2010

[5] M. de Vos, A. W. Gunst, R. Nijboer, "The LOFAR Telescope: System Architecture and Signal Processing”, Proceedings of the IEEE, Vol. 97, No. 8, August 2009

[6] C. J. Lonsdale et.al., “The Murchison Widefield Array: Design Overview”, Proceedings of the IEEE, Vol. 97, No. 8, August 2009 